\title{
TOPOLOGICAL CONJUGACY AND TRANSITIVITY FOR A CLASS OF PIECEWISE MONOTONE MAPS OF THE INTERVAL
}

\author{
LOUIS BLOCK AND ETHAN M. COVEN
}

\begin{abstract}
We say that a continuous map $f$ of a compact interval to itself is linear Markov if it is piecewise linear, and the set of all $f^{k}(x)$, where $k \geq 0$ and $x$ is an endpoint of a linear piece, is finite. We provide an effective classification, up to topological conjugacy, for linear Markov maps and an effective procedure for determining whether such a map is transitive. We also consider expanding Markov maps, partly to motivate the proof of the more complicated linear Markov case.
\end{abstract}

1. Introduction. The basic equivalence relation of dynamical systems is topological conjugacy. Two continuous maps $f: X \rightarrow X$ and $g: Y \rightarrow Y$ of topological spaces to themselves are called topologically conjugate if there exists a homeomorphism $h$ of $X$ onto $Y$ (called a (topological) conjugacy) such that $g=h \circ f \circ h^{-1}$. In this paper we give an effective classification, up to topological conjugacy, for a class of continuous, piecewise monotone maps of the interval, as well as an effective procedure for determining whether a map in the class is (topologically) transitive.

We say a continuous map $f$ of a compact interval to itself is linear Markov if it is piecewise linear, and the set $P$ of all $f^{k}(x)$, where $k \geq 0$ and $x$ is an endpoint of a linear piece, is finite. In $\S 2$, we associate with $f$ a map $f^{*}: P^{*} \rightarrow P^{*}$, where $P^{*}$ is the indexing set of a certain $f$-invariant subset of $P$. The main result of this paper is the following.

THEOREM 2.6. Linear Markov maps $f$ and $g$ are topologically conjugate if and only if $f^{*}=g^{*}$ or $f^{*}=^{*} g$.

Here, if $P^{*}=\{1, \ldots, N\}$, then ${ }^{*} f: P^{*} \rightarrow P^{*}$ is the reverse of $f^{*}$, defined by ${ }^{*} f(i)=N+1-f^{*}(N+1-i)$. In the theorem, $f^{*}=g^{*}$ or ${ }^{*} g$ according as the conjugacy is order-preserving or order-reversing.

Since $f^{*}$ depends only on the action of $f$ on the finite set $P$, the classification is effective.

Conversely, a map $\varphi$ of a finite set of at least two real numbers into (not necessarily onto) itself determines a linear Markov map $f$ by "connecting the dots": $f \mid P=\varphi$ and $f$ is linear between adjacent points of $P$. Maps of the interval obtained in this way have been used to provide models of various types of dynamical behavior. The maps constructed by P. Stefan $[\mathbf{S t}]$ to show that the Sarkovskii ordering is sharp are of this form. For example, if $\varphi(1,2,3,4,5)=(3,5,4,2,1)$, then

Received by the editors February 21, 1985 and, in revised form, February 25, 1986. Presented to the Society, May 3, 1985, Mobile, Alabama.

1980 Mathematics Subject Classification (1985 Revision). Primary 54H20, 58F15.

This work was done while the first author was J. H. Van Vleck Visiting Professor at Wesleyan University. 
the linear Markov map determined by $\varphi$ has periodic points of all periods except 3. (Here, and in all subsequent examples, we use $\varphi\left(x_{1}, \ldots, x_{n}\right)=\left(y_{1}, \ldots, y_{n}\right)$ to denote $P=\left\{x_{1}<\cdots<x_{n}\right\}$ and $\varphi\left(x_{i}\right)=y_{i}$.) A piecewise monotone map of the interval having points of periods $2^{n}(n=0,1,2, \ldots)$ but of no other periods may be obtained as a limit of maps of this form $[\mathbf{N}]$. These maps also arise in the study of smooth maps of the interval; e.g., P. Stein and S. Ulam [SU] showed that the linear Markov map determined by $\varphi(0,1 / 2,1)=(0,1,0)$ is topologically conjugate to the quadratic map $x \mapsto 4 x(1-x)$ of $[0,1]$ to itself. The structure of the periodic orbits of linear Markov maps determined by permutations has been studied by $\mathrm{C}$. Bernhardt $[\mathbf{B e}-\mathbf{1}, \mathbf{2}]$.

To motivate the proof of Theorem 2.6, we first prove the analogous result in a simpler setting. We say a continuous, piecewise monotone map $f$ is expanding Markov if the set $P$ of all $f^{k}(x)$, where $k \geq 0$ and $x$ is an endpoint of a monotone piece, is finite, and there is a constant $c>1$ such that $|f(x)-f(y)| \geq c|x-y|$ whenever $x$ and $y$ lie in the same monotone piece. If $f$ is expanding Markov and $P=\left\{x_{1}<\cdots<x_{N}\right\}$, we let $P^{*}=\{1, \ldots, N\}$ and define $f^{*}: P^{*} \rightarrow P^{*}$ by $f^{*}(i)=j$ if $f\left(x_{i}\right)=x_{j}$. With this notation, we prove in Theorem 2.1 that Theorem 2.6 holds for expanding Markov maps. A special case of Theorem 2.1 was obtained by H. Rüssmann and E. Zehnder [RZ]. The invariant measures of not-necessarilycontinuous linear and expanding Markov maps have been studied by W. Byers and A. Boyarsky [BB].

Let $\mathcal{M}$ denote the set of maps which are either linear Markov or expanding Markov. We remark that if $f$ is both linear Markov and expanding Markov, then the two definitions of $f^{*}: P^{*} \rightarrow P^{*}$ coincide. We will see in Theorem 2.7 that Theorem 2.6 holds for maps in $\mathcal{M}$, and in Corollary 2.8 that $f \in \mathcal{M}$ is topologically conjugate to the linear Markov map determined by $f^{*}$. Thus we have a canonical representative (actually two) of each conjugacy class in $\mathcal{M}$.

In $\S 3$ we look at transitivity. We associate with each map in $\mathcal{M}$ a $0-1$ matrix and show in Theorem 3.1 that the map is transitive if and only if the matrix is irreducible but not a permutation matrix. It follows from Theorem 3.1 that the maps constructed by Stefan having points of a given odd period greater than one but of no smaller odd period greater than one are transitive, but that those having no points of odd period greater than one are not transitive. We give an example of a map $\varphi$ which determines a transitive linear Markov map having points of period 6 but none of odd period greater than one. We show that, more generally, any continuous (piecewise monotone or not), transitive map of the interval must have a point of period 6 . We do this by showing that if such an $f$ is transitive, then $f^{2}$ is turbulent $[\mathbf{B C}]$.

We also give a short proof, for the special case $f \in \mathcal{M}$, of a theorem of W. Parry $[\mathbf{P}]$ which states that any continuous, transitive, piecewise monotone map $f$ of the interval is topologically conjugate to a piecewise linear map $g$ which has slope $\pm \beta$ $(\log \beta=$ topological entropy of $f)$ on each linear piece. We show that $g$ is essentially unique.

The authors thank Mike Boyle and Bruce Kitchens for helpful conversations, especially in regard to Theorem 3.2 .

2. Classification. In this section, we prove the main result of the paper, the Classification Theorem for linear Markov maps (Theorem 2.6). 
We first prove Theorem 2.1, the Classification Theorem for expanding Markov maps. The straightforward construction in this proof provides a model for the more complicated construction needed to deal with linear Markov maps in Theorem 2.6.

Let $f$ and $g$ be expanding Markov maps. Recall that $P=\left\{x_{1}<\cdots<x_{N}\right\}$ is the set of all $f^{k}(x)$, where $k \geq 0$ and $x$ is an endpoint of a monotone piece, $P^{*}=\{1, \ldots, N\}$, and $f^{*}: P^{*} \rightarrow P^{*}$ is defined by $f^{*}(i)=j$ if $f\left(x_{i}\right)=x_{j}$. Let $Q=\left\{y_{1}<\cdots<y_{M}\right\}$ and $g^{*}: Q^{*} \rightarrow Q^{*}$ be the corresponding objects for $g$.

THEOREM 2.1. Expanding Markov maps $f$ and $g$ are topologically conjugate via an order-preserving homeomorphism if and only if $f^{*}=g^{*}$.

PROOF. We leave it to the reader to show that if $f$ and $g$ are topologically conjugate via an order-preserving homeomorphism, then $f^{*}=g^{*}$.

Assuming $f^{*}=g^{*}$, we construct an order-preserving conjugacy as follows. For $0 \leq n \leq \infty$, let $P_{n}=\bigcup_{k=0}^{n} f^{-k}(P)$ and $Q_{n}=\bigcup_{k=0}^{n} g^{-k}(Q)$. Since $f$ and $g$ are expanding, $P_{\infty}$ is dense in $\left[x_{1}, x_{N}\right]$ and $Q_{\infty}$ is dense in $\left[y_{1}, y_{N}\right]$. For $0 \leq n<\infty$, we define order-preserving maps $h_{n}$ of $P_{n}$ onto $Q_{n}$ such that $h_{n}=h_{n+1} \mid P_{n}$ and $g \circ h_{n}=h_{n} \circ\left(f \mid P_{n}\right)$. We then define $h_{\infty}: P_{\infty} \rightarrow Q_{\infty}$ by $h_{\infty}(x)=h_{n}(x)$ if $x \in P_{n}$. Thus $h_{\infty}$ is a well-defined order-preserving map of $P_{\infty}$ onto $Q_{\infty}$, and hence extends to an order-preserving homeomorphism $h$ of $\overline{P_{\infty}}=\left[x_{1}, x_{N}\right]$ onto $\overline{Q_{\infty}}=\left[y_{1}, y_{N}\right]$. Since $h_{\infty} \circ f \mid P_{\infty}=g \circ h_{\infty}$, it follows that $h \circ f=g \circ h$.

The constructions are simple. Define $h_{0}$ on $P_{0}$ by $h_{0}\left(x_{i}\right)=y_{i}$. It is easy to see that $P_{1}$ and $Q_{1}$ have the same number of points. Writing $P_{1}=\left\{z_{i}\right\}$ and $Q_{1}=\left\{w_{i}\right\}$ in increasing order, we have $f\left(z_{i}\right)=z_{j}$ if and only if $g\left(w_{i}\right)=w_{j}$, and $z_{i} \in P_{0}$ if and only if $w_{i} \in Q_{0}$. Define $h_{1}$ on $P_{1}$ by $h_{1}\left(z_{i}\right)=w_{i}$. Continue inductively.

The expanding property was used only to make $\bigcup f^{-n}(P)$ dense. Thus Theorem 2.1 holds for maps for which some power is expanding Markov.

For linear Markov maps, we cannot just copy the proof of Theorem 2.1, because $\bigcup f^{-n}(P)$ need not be dense. (Consider the identity map. For nontrivial examples, see (2) and (3) at the end of this section.) For such maps we construct an invariant set $\mathbf{P}$, consisting of finitely many points and finitely many closed inversals, which will play the role of $P$.

Let $f$ be a linear Markov map. Recall that $P$ is the set of all $f^{k}(x)$, where $k \geq 0$ and $x$ is an endpoint of a linear piece. Let $J$ be a nondegenerate closed interval whose endpoints are in $P$. We say that $J$ is periodic if for some $n>0, f^{n}(x)=x$ for all $x \in J$; and $J$ is flat if $f(x)=f(y)$ for all $x, y \in J$.

LEMMA 2.2. If $J$ is a maximal periodic interval, then the endpoints of $J$ have the same period.

PROOF. Let the endpoints of $J$ be $x<y$. We assume that $x$ and $y$ are in different orbits: otherwise there is nothing to prove. It suffices to show that $f^{k}(y)=y$ whenever $f^{k}(x)=x$.

Suppose that $f^{k}(x)=x$ but $f^{k}(y) \neq y$. There are three possibilities:

(a) $x<f^{k}(y)<y$

(b) $y<f^{k}(y)$

(c) $f^{k}(y)<x$.

Suppose (a) holds. Then $f^{k}(J)$ is a proper subset of $J$, and hence $J$ cannot consist entirely of periodic points. 
Suppose (b) holds. Since $f^{k}$ is monotone on $f^{k}(J), f^{k}(y)<f^{2 k}(y)$. It follows by induction that $y<f^{i k}(y)$ for every $i>0$, contradicting the periodicity of $y$.

Suppose (c) holds. If $f^{2 k}(y)<x$, we obtain a contradiction as in (a) or (b), using $f^{k}(J)$ in place of $J$. If $x<f^{2 k}(y)<y$ or $y<f^{2 k}(y)$, we obtain a contradiction as in (a) or (b), using $f^{2 k}$ in place of $f^{k}$. Suppose then that $f^{2 k}(y)=y$. Then $J \cup f^{k}(J)$ is a periodic interval, contradicting the maximality of $J$.

LEMMA 2.3. Let $J$ be a closed interval whose endpoints are fixed by $f^{n}$ and such that $f^{n}$ is strictly monotone on $J$. Then $f^{n}$ is the identity on $J$, and $J$ is contained in a maximal periodic interval.

ProOF. Since $f^{n}$ is piecewise linear on $J$, to show that it is the identity on $J$ it suffices to show that $f^{n}(x)=x$ for every $x \in J$ which is an endpoint of a linear piece of $f^{n}$. Any such $x$ must be in $\bigcup_{k=0}^{n-1} f^{-k}(P)$; thus $f^{n}(x) \in P$ and $x$ has a finite orbit. Since $f^{n}$ is increasing, $f^{n}(x)=x$.

Now let $K$ be the largest closed interval containing $J$ such that $f^{n}$ is the identity on $K$. It follows as in the paragraph above that the endpoints of $K$ are in $P$. Thus $K$ must be a maximal periodic interval.

We call a maximal periodic interval orientation-preserving or orientationreversing according as its endpoints are in different orbits or the same orbit. Lemma 2.4 justifies this terminology.

LEMMA 2.4. Let $J$ be a maximal periodic interval and let $n$ be the common period of the endpoints of $J$.

(1) If $J$ is orientation-preserving, then the intervals $J, f(J), \ldots, f^{n-1}(J)$ are pairwise disjoint.

(2) If $J$ is orientation-reversing, then $n$ is even, the intervals $J, f(J), \ldots$, $f^{n / 2-1}(J)$ are pairwise disjoint, and there is a unique fixed point $\bar{x}$ of $f^{n / 2}$ in $J$. Furthermore, if $J^{\prime}=\{x \in J \mid x \leq \bar{x}\}$, then $J^{\prime} \cup f^{n / 2}\left(J^{\prime}\right)=J$ and the intervals $J^{\prime}, f\left(J^{\prime}\right), \ldots, f^{n-1}\left(J^{\prime}\right)$ are almost pairwise disjoint: $f^{k}\left(J^{\prime}\right) \cap f^{k+n / 2}\left(J^{\prime}\right)=\left\{f^{k}(\bar{x})\right\}$ for $0 \leq k \leq n / 2-1$.

PROOF. Since $f^{n}(J)=J$, if the intervals $J, f(J), \ldots, f^{n-1}(J)$ are not pairwise disjoint, then $J \cap f^{k}(J) \neq \varnothing$ for some $k, 1 \leq k \leq n-1$. Since $f^{k}(J)$ is also periodic, so too is $J \cup f^{k}(J)$. Hence by the maximality of $J, f^{k}(J) \subseteq J$, and we have $J=f^{k n}(J) \subseteq f^{k}(J) \subseteq J$. Therefore $f^{k}(J)=J$. Since $k<n$, the endpoints of $J$ must be interchanged by $f^{k}$, and so $k=n / 2$ and $J$ is orientation-reversing. Then $f^{n / 2}$ has a unique fixed point $\bar{x} \in J$. The remainder of (2) now follows.

Let $P^{\prime}=\left\{x_{1}<\cdots<x_{N}\right\}$ be the set of all $f^{k}(x)$, where $k \geq 0$ and $x$ is one of the following: an endpoint of a monotone piece, an endpoint of a maximal periodic interval, or an endpoint of a maximal flat interval.

Let

$$
\mathbf{P}=P^{\prime} \cup \bigcup J
$$

where the union is taken over all maximal periodic and flat intervals.

LEMMA 2.5. $\bigcup_{n=0}^{\infty} f^{-n}(\mathbf{P})$ is dense. 
ProOF. Let $K$ denote the collection of closed intervals whose endpoints are adjacent members of $P$. Write $K=K_{0} \cup K_{1} \cup K_{2}$ where

$$
\begin{aligned}
& \mathcal{K}_{0}=\{K \mid f(K) \text { is a point }\}, \\
& \mathcal{K}_{1}=\{K \mid f(K) \in \mathcal{K}\}, \\
& \mathcal{K}_{2}=\{K \mid f(K) \text { is a union of at least two members of } K\} .
\end{aligned}
$$

For any interval $J$, let $|J|$ denote the length of $J$, and let

$$
c=\min \frac{|f(K)|}{\left|K^{\prime}\right|},
$$

where the minimum is taken over all pairs $\left(K, K^{\prime}\right)$ with $K \in \mathcal{K}_{2}, K^{\prime} \in \mathcal{K}$, and $f(K) \supseteq K^{\prime}$. Note that $c>1$.

To prove the lemma, it suffices to show that if $J$ is a closed interval such that for every $n \geq 0, f^{n}(J)$ contains no turning points and meets no flat intervals, then for some $n \geq 0, f^{n}(J)$ is contained in a maximal periodic interval. Let $J$ be such an interval.

Claim 1. For some $n \geq 0$, either $f^{n}(J) \cap P \neq \varnothing$ or $f^{n}(J)$ is contained in a maximal periodic interval.

To prove the claim, suppose $f^{n}(J) \cap P=\varnothing$ for every $n \geq 0$. Then for every $n \geq 0$, there exists $K_{n} \in K_{1} \cup K_{2}$ such that $f^{n}(J) \subseteq K_{n}$. If $K_{n} \in K_{1}$, then since $f$ is linear on $K_{n},\left|f^{n+1}(J)\right| /\left|f^{n}(J)\right|=\left|K_{n+1}\right| /\left|K_{n}\right|$, i.e., $\left|f^{n+1}(J)\right| /\left|K_{n+1}\right|=\left|f^{n}(J)\right| /\left|K_{n}\right|$. If $K_{n} \in K_{2}$, then $\left|f^{n+1}(J)\right| /\left|K_{n+1}\right| \geq c\left|f^{n}(J)\right| /\left|K_{n}\right|$. Since $f^{n}(J) \cap P=\varnothing$ for every $n \geq 0$, it follows that $K_{n} \in K_{1}$ for all but finitely many $n$, say all $n \geq m$. Then $f\left(K_{m}\right)=K_{m+1}, f\left(K_{m+1}\right)=K_{m+2}$, etc., and $f$ is a homeomorphism on each of these intervals. Hence for some $i \geq 0$ and $j>0, K_{m+i}=K_{m+i+j}$, and thus $K_{m+i}$ satisfies the hypotheses of Lemma 2.3 (with $n=2 j$ ). Therefore $K_{m+i}$ and hence $f^{m+i}(J)$ are contained in a maximal periodic interval. The claim is proved.

Suppose the first conclusion of claim 1 holds (otherwise the proof is complete). We may assume that one endpoint of $J$ is in $P$. In the same manner as claim 1 , we have

Claim 2. For some $n \geq 0$, either $f^{n}(J)$ contains two members of $P$ or is contained in a maximal periodic interval.

Now we may assume that $J$ is a union of members of $K$, and hence so is $f^{n}(J)$ for every $n \geq 0$. Since there are only finitely many different such unions, there exist $i \geq 0$ and $j>0$ such that $f^{i}(J)=f^{i+j}(J)$. It follows from Lemma 2.3 that $f^{i}(J)$ is contained in a maximal periodic interval.

With $P^{\prime}=\left\{x_{1}<\cdots<x_{N}\right\}$ as above, let $P^{*}=\{1, \ldots, N\}$, and define $f^{*}: P^{*} \rightarrow$ $P^{*}$ by $f^{*}(i)=j$ if $f\left(x_{i}\right)=x_{j}$.

THEOREM 2.6. Linear Markov maps $f$ and $g$ are topologically conjugate if and only if $f^{*}=g^{*}$ or $f^{*}={ }^{*} g$.

PROOF. Again we prove only that if $f^{*}=g^{*}$, then $f$ and $g$ are topologically conjugate.

Let $\mathbf{P}$ be the object described before Lemma 2.5 for $f$, and let $\mathbf{Q}$ be the corresponding object for $g$. The proof proceeds as the proof of Theorem 2.1, with $\mathbf{P}$ and $\mathbf{Q}$ playing the roles of $P$ and $Q$. Thus, using Lemma 2.5, it suffices to define 
order-preserving maps $h_{n}: \mathbf{P}_{n} \rightarrow \mathbf{Q}_{n}$ for $0 \leq n<\infty$ such that $h_{n}=h_{n+1} \mid \mathbf{P}_{n}$ and $g \circ h_{n}=h_{n} \circ\left(f \mid \mathbf{P}_{n}\right)$.

Define $h_{0}$ on $P^{\prime}$ by $h_{0}\left(x_{i}\right)=y_{i}$.

Extend $h_{0}$ to maximal flat intervals, one such interval at a time, as follows. If $J=$ $\left[x_{i}, x_{j}\right]$ is such an interval, then so is $K=\left[y_{i}, y_{j}\right]$. Let $h_{0} \mid J$ be any homeomorphism of $J$ onto $K$ for which $h_{0}\left(x_{k}\right)=y_{k}, i \leq k \leq j$.

Extending $h_{0}$ to maximal periodic intervals is more complicated. We do it one orbit of such intervals at a time, and the procedure is slightly different for orientation-preserving and orientation-reversing intervals.

Suppose $J=\left[x_{i}, x_{j}\right]$ is an orientation-preserving maximal periodic interval, and let $n$ be the common period of $x_{i}$ and $x_{j}$. Then the same holds for $K=\left[y_{i}, y_{j}\right]$. Let $h_{0} \mid J$ to be any homeomorphism of $J$ onto $K$ for which $h_{0}\left(x_{k}\right)=y_{k}, i \leq k \leq j$. By Lemma 2.4, the intervals $J, f(J), \ldots, f^{n-1}(J)$ are pairwise disjoint, and $f$ is a homeomorphism on each of them. (The analogous statements hold for $K$ and $g$.) Let $h_{0} \mid f(J)=g \circ h_{0} \circ(f \mid J)^{-1}$. Since $f$ is order-preserving on $J$ if and only if $g$ is order-preserving on $K$, it follows that $h_{0}$ is order-preserving. Continue this process around the orbit of $J$. By Lemma $2.3, f^{n}$ is the identity on $J$, so $h_{0}$ is well defined on this orbit, and on it $h_{0} \circ f=g \circ h_{0}$.

Suppose $J=\left[x_{i}, x_{j}\right]$ is an orientation-reversing maximal periodic interval and again let $n$ be the common period of $x_{i}$ and $x_{j}$. Let $\bar{x}$ be the unique fixed point of $f^{n / 2}$ in $J$ and $\bar{y}$ the unique fixed point of $g^{n / 2}$ in $K=\left[y_{i}, y_{j}\right]$. Let $J^{\prime}=$ $\left[x_{i}, \bar{x}\right]$ and let $h_{0} \mid J^{\prime}$ be any homeomorphism of $J^{\prime}$ onto $K^{\prime}=\left[y_{i}, \bar{y}\right]$ for which $h_{0}(\bar{x})=\bar{y}$ and $h_{0}\left(x_{k}\right)=y_{k}$ whenever $x_{k} \in P^{\prime}$ and $x_{i} \leq x_{k} \leq \bar{x}$. The intervals $J^{\prime}, f\left(J^{\prime}\right), \ldots, f^{n-1}\left(J^{\prime}\right)$ are almost pairwise disjoint: $f^{k}\left(J^{\prime}\right) \cap f^{k+n / 2}\left(J^{\prime}\right)=\left\{f^{k}(\bar{x})\right\}$, $0 \leq k \leq n / 2-1$, are the only nonempty intersections. Thus $h_{0}$ can be extended around the orbit of $J^{\prime}$ as in the orientation-preserving case. Since $\bigcup_{k=0}^{n-1} f^{k}\left(J^{\prime}\right)=$ $\bigcup_{k=0}^{n / 2-1} f^{k}(J)$, we have extended $h_{0}$ around the orbit of $J$.

Doing this procedure on each orbit of maximal periodic intervals defines $h_{0}$ on $\mathbf{P}_{0}$ with the desired properties.

In order to avoid notational clutter, we construct only $h_{1}: \mathbf{P}_{1} \rightarrow \mathbf{Q}_{1}$ as a model of the inductive step. Since $f^{*}=g^{*}, P^{\prime} \cup f^{-1}\left(P^{\prime}\right)$ and $Q^{\prime} \cup g^{-1}\left(Q^{\prime}\right)$ have the same number of points. Writing $P^{\prime} \cup f^{-1}\left(P^{\prime}\right)=\left\{z_{i}\right\}$ and $Q^{\prime} \cup g^{-1}\left(Q^{\prime}\right)=\left\{w_{i}\right\}$ in increasing order, we have $z_{i} \in P^{\prime}$ if and only if $w_{i} \in Q^{\prime}$, and $f\left(z_{i}\right)=z_{j}$ if and only if $g\left(w_{i}\right)=w_{j}$. Furthermore,

$$
\mathbf{P}_{1}=\mathbf{P}_{0} \cup f^{-1}\left(P^{\prime}\right) \cup \bigcup J
$$

where the union is taken over all closed intervals $J$ maximal with respect to the following properties: $J^{0} \cap \mathbf{P}_{0}=\varnothing$ and $f(J)$ is contained in a component of $\mathbf{P}_{0}$. Note that the endpoints of any such $J$ are in $f^{-1}\left(P^{\prime}\right)$. (Analogous statements hold for $\mathbf{Q}_{1}$.)

Define $h_{1}$ on $P^{\prime} \cup f^{-1}\left(P^{\prime}\right)$ by $h_{1}\left(z_{i}\right)=w_{i}$.

Extend $h_{1}$ to the intervals described above, one at a time, as follows. If $J=$ $\left[z_{i}, z_{j}\right]$ is such an interval, then so is $K=\left[w_{i}, w_{j}\right]$, and $f$ is a homeomorphism on $J$, as is $g$ on $K$. Let $h_{1} \mid J=(g \mid K)^{-1} \circ h_{0} \circ f$. Doing this for each such $J$ produces a map $h_{1}$ with the desired properties.

Let $f$ be a linear Markov map and let $P$ be as above. If $f$ is also expanding Markov, then there can be no periodic or flat intervals, and therefore $\mathbf{P}=P^{\prime}$ is the 
set of all $f^{k}(x)$, where $k \geq 0$ and $x$ is an endpoint of a monotone piece. Thus $P^{*}$ and $f^{*}$ are well defined, and we obtain

THEOREM 2.7. $f, g \in \mathcal{M}$ are topologically conjugate if and only if $f^{*}=g^{*}$ or $f^{*}={ }^{*} g$.

Suppose that $\varphi: P \rightarrow P$ is a map of a finite set of at least two real numbers into (not necessarily onto) itself. We say that $\varphi$ determines the linear Markov map $f$ obtained by "connecting the dots": $f \mid P=\varphi$ and $f$ is linear between adjacent points of $P$.

COROLLARY 2.8. $f \in \mathcal{M}$ is topologically conjugate to the linear Markov map determined by $f^{*}$.

Thus we have two canonical representatives, $f^{*}$ and ${ }^{*} f$, of each conjugacy class.

We conclude this section with three examples.

The reader who works through these examples is reminded that in an orientationpreserving maximal periodic interval, all points have the same period, while in an orientation-reversing maximal periodic interval, all points have the same period except for the unique "central" point.

(1) The linear Markov maps determined by

(a) $\varphi(1,2,3,6,7)=(2,7,3,2,1)$,

(b) $\varphi(1,2,6,7,8)=(2,8,7,2,1)$, and

(c) $\varphi(1,2,3,4,5,8,9)=(3,5,9,8,4,2,1)$ are topologically conjugate, and $f^{*}(1,2,3)=(2,3,1)$.

(2) The linear Markov maps determined by

(a) $\varphi(1,2,3,4,5,8,9)=(4,5,8,9,3,2,1)$,

(b) $\varphi(1,2,7,8,10,15,17,19,20)=(8,17,19,20,19,17,7,2,1)$, and

(c) $\varphi(1,2,4,5,7,8,9,15,16,17,18,24,29,30)=(8,17,18,24,29,30,24,18,8,7$, $5,4,2,1)$ are topologically conjugate, and $f^{*}(1,2,3,4,5,6,7)=(4,5,6,7,3,2,1)$.

(3) The linear Markov maps determined by

(a) $\varphi(1,3,4,5,6,8,9,12,13,15,16,20,25,27,29,30)=(8,9,12,13,15,30,29,27$, $25,6,1,12,5,4,3,1)$,

(b) $\varphi$ as in (a) except $\varphi(20)=9$, and

(c) $\varphi$ as in (b) except $\varphi(6)=29$ and $\varphi(15)=15$

are topologically conjugate and

$$
f^{*}(1,2,3,4,5,6,7,8,9,10,11)=(4,5,6,11,10,9,1,5,3,2,1) .
$$

Note that in (2) the maximal periodic intervals are orientation-reversing, while in (3) they are orientation-preserving. (3) also illustrates the fact that if $x$ is a turning point and $f(x)$ is in the interior of an orientation-preserving maximal periodic interval, then the conjugacy class of $f$ is not affected by moving $f(x)$ to another point in that interior, provided that the orbit of no other turning point meets the interior.

3. Transitivity. A continuous map $f$ of a compact metric space $X$ to itself is transitive if $f$ maps $X$ onto $X$ and there exists a point with a dense orbit, or equivalently, if the only closed invariant set $E$ with $E^{\circ} \neq \varnothing$ is $E=X$. For maps 
of the interval, we shall use this in the following form: $f$ is transitive if and only if for every closed interval $J, \bigcup_{n>0} f^{n}(J)$ is dense.

We associate with $f \in \mathcal{M}$ a $0-1$ matrix $A$ as follows. If $F$ is the linear Markov map determined by $f^{*}: P^{*} \rightarrow P^{*}=\{1, \ldots, N\}$, then $A$ is the $(N-1) \times(N-1)$ matrix such that $A(i, j)=1$ if $F[i, i+1] \supseteq[j, j+1]$, and $A(i, j)=0$ if not.

Recall that a nonnegative matrix $B$ is irreducible if for every $i, j$ there exists $n$ such that $B^{n}(i, j)>0$.

THEOREM 3.1. Let $f \in \mathcal{M}$ and let $A$ be as above. Then $f$ is transitive if and only if $A$ is irreducible but not a permutation matrix.

PROOF. We omit the straightforward proof that $A$ is irreducible but not a permutation matrix whenever $f$ is transitive.

To prove the other implication, we may assume that $f$ is the linear Markov map determined by $f^{*}$. Let $J$ be a closed interval contained in $[1, N]$. By arguments similar to those used in the proof of Lemma 2.5, it follows that for some $n, f^{n}(J)$ contains an interval $[i, i+1]$. But $A$ is irreducible, so for each $k=1,2, \ldots, N_{-1}$, there exists $m$ such that $f^{m}[i, i+1] \supseteq[k, k+1]$. Thus $f$ is transitive.

A continuous map $f: I \rightarrow I$ of the interval is strongly transitive $[\mathbf{P}]$ if for every nondegenerate interval $J \subseteq I$, there exists $N$ such that $\bigcup_{k=0}^{N} f^{k}(J)=I$. Every continuous, transitive, piecewise monotone map is strongly transitive $[\mathbf{C M}]$. According to a theorem of Parry $[\mathbf{P}]$, every continuous, (strongly) transitive, piecewise monotone map $f$ is topologically conjugate to a piecewise linear map whose linear pieces have slopes $\pm \beta$, where $\log \beta$ is the topological entropy of $f$. (It follows from [MS] that $\beta$ is the largest eigenvalue of $A$.) Let us call such a map a Parry map. The existence part of the following theorem is of course a special case of Parry's Theorem.

THEOREM 3.2. Let $f \in \mathcal{M}$ be transitive. Then, up to scale and orientation, there is exactly one Parry map which is topologically conjugate to $f$.

PROOF. We may assume again that $f$ is the linear Markov map determined by $f^{*}: P^{*} \rightarrow P^{*}=\{1, \ldots, N\}$. Let $A$ be as above. By Theorem 3.1, $A$ is irreducible; hence by the Frobenius-Perron Theorem $[\mathbf{G}]$, there is unique positive vector $l=\left(l_{i}\right)$ which satisfies $\sum l_{i}=N-1$ and $A l=\beta l$, where $\beta$ is the largest eigenvalue of $A$.

Let $y_{1}=1$ and for $i \geq 2$, let $y_{i}=l_{1}+\cdots+l_{i-1}$. Define $g:[1, N] \rightarrow[1, N]$ by $g\left(y_{i}\right)=y_{j}$ if $f\left(x_{i}\right)=x_{j}$, and linearly between each $y_{i}$ and $y_{i+1}$. By Theorem 2.6, $f$ and $g$ are topologically conjugate.

To prove uniqueness, suppose $h:[1, N] \rightarrow[1, N]$ is a Parry map topologically conjugate to $g$ via an orientation-preserving homeomorphism, and let $Q=\left\{y_{1}<\right.$ $\left.\cdots<y_{N}\right\}$ be associated with $h$ as in $\S 2$. Let $k=\left(k_{i}\right)$, where $k_{i}=y_{i+1}-y_{i}$. Since $h$ is a Parry map, $A k=\beta k$, and since $h$ is defined on $[1, N], \sum k_{i}=N-1$. Hence $k=l$ and $h=g$.

We now turn to some more examples. Recall the Sarkovskir ordering of the positive integers:

$$
3 \triangleleft 5 \triangleleft 7 \triangleleft \cdots \triangleleft 2 \cdot 3 \triangleleft 2 \cdot 5 \triangleleft \cdots \triangleleft 2^{2} \cdot 3 \triangleleft 2^{2} \cdot 5 \triangleleft \cdots \triangleleft 2^{2} \triangleleft 2^{1} \triangleleft 2^{0} .
$$

Sarkovskiu's Theorem [Sa, $\mathbf{S t}$ ] states that if $f$ has a point of period $n$, then for every $k \triangleright n f$ also has a point of period $k$. In $[\mathbf{S t}]$ P. Stefan constructed for each $n$ 
a linear Markov map $f_{n}$ which has periodic points of period $n$ but none of period $k$ for any $k \triangleleft n$. $f_{n}$ is determined by a map $\varphi_{n}$, which is constructed as follows. $\varphi_{1}(1,2)=(1,2)$, and for odd $n>1$,

$$
\begin{aligned}
\varphi_{3}(1,2,3) & =(2,3,1), \\
\varphi_{5}(1,2,3,4,5) & =(3,5,4,2,1), \\
\varphi_{7}(1,2,3,4,5,6,7) & =(4,7,6,5,3,2,1),
\end{aligned}
$$

and the obvious pattern continues. For even $n$, the "square-root trick" is used inductively: $\varphi_{2 n}=\sqrt{\varphi_{n}}$, where the square root is defined as follows. If $\varphi:\{1, \ldots, k\} \rightarrow$ $\{1, \ldots, k\}$, then $\sqrt{\varphi}:\{1, \ldots, 2 k\} \rightarrow\{1, \ldots, 2 k\}$, where

$$
\sqrt{\varphi}(i)= \begin{cases}\varphi(i)+k & \text { if } 1 \leq i \leq k, \\ i-k & \text { if } k+1 \leq i \leq 2 k .\end{cases}
$$

(Note that $(\sqrt{\varphi} \mid\{1, \ldots, k\})^{2}=\varphi$.)

By Theorem 3.1, $f_{n}$ is transitive if and only if $n$ is odd and greater than one.

Consider the linear Markov map $f$ determined by $\varphi(1,2,3,4,5)=(3,5,3,1,3)$. It is easy to see that $f$ has periodic points of period 6 , but none of odd period greater than one. By Theorem 3.1, $f$ is transitive.

Corollary 3.5 below states that for $n \triangleright 6$ there can be no continuous (piecewise monotone or not), transitive map of the interval which has periodic points of period $n$ but none of period $k$ for any $k \triangleleft n$.

A continuous map $f$ of the interval is called turbulent $[\mathbf{B C}]$ if there are closed intervals $J$ and $K$, with at most one point in common, such that $f(J) \cap f(K) \supseteq$ $J \cup K$.

LEMMA 3.3. A transitive map which is not turublent has a unique fixed point. The fixed point is not an endpoint.

ProOF. Suppose $f: I \rightarrow I$ is transitive but not turbulent and has fixed points $a<b$. Without loss of generality, there are no fixed points in $(a, b)$ and $f(x)>x$ for every $x \in(a, b)$. Since $f$ is transitive, $f(d)=a$ for some least $d>a$ and $f(c)=d$ for some least $c \in(a, d)$. (If there were no such $c$, then $[a, d]$ would be invariant and hence equal to $I$. But then $f$ would not be onto.) In this case $J=[a, c]$ and $K=[c, d]$ show that $f$ is turbulent. Thus $f$ has exactly one fixed point; call it $e$. If $e$ were an endpoint of $I$, say the left endpoint, then $f(x)<x$ for every $x \neq e$ and again $f$ would not be onto.

THEOREM 3.4. Let $f$ be a continuous map of the interval. If $f$ is transitive, then $f^{2}$ is turbulent.

PROOF. Let $f: I \rightarrow I$. If $f^{2}$ is transitive, then $f$ has a point of odd period greater than one $[\mathbf{B M}-2]$ and so $[\mathbf{B C}] f^{2}$ is turbulent. If $f^{2}$ is not transitive, then $I=J \cup K$, where $J$ and $K$ are closed $f^{2}$-invariant intervals with one point (which must be a fixed point of $f$ ) in common and such that $f^{2} \mid J$ and $f^{2} \mid K$ are transitive [BM-1]. By Lemma 3.3, $f^{2} \mid J$ and hence $f^{2}$ is turbulent. 
Since turbulent maps have periodic points of period 3 and topological entropy at least $\log 2[\mathbf{B C}]$, we have

COROLlaRY 3.5. If $f$ is transitive, then $f$ has a periodic point of period 6 .

COROLlaRY $3.6[\mathbf{B l}]$. If $f$ is transitive, then the topological entropy of $f$ is at least $\log \sqrt{2}$.

\section{REFERENCES}

[BB] W. Byers and A. Boyarsky, Absolutely continuous invariant measures that are maximal, Trans. Amer. Math. Soc. 290 (1985), 303-314.

[BM-1] M. Barge and J. Martin, Chaos, periodicity, and snakeline continua, Trans. Amer. Math. Soc. 289 (1986), 355-365.

[BM-2] _ Dense orbits on the interval, preprint, 1984.

[Be-1] C. Bernhardt, Simple permutations with order a power of two, Ergodic Theory Dynamical Systems 4 (1984), 179-186.

[Be-2] _ Oriented Markov graphs of the interval, preprint, 1984.

[BC] L. Bloch and A. Coppel, Stratification of continuous maps of an interval, Trans. Amer. Math. Soc. 297 (1986), 587-604.

[B1] A. Blokh, On sensitive mappings of the interval, Russian Math. Surveys 37 (1982), 203204.

[CM] E. Coven and I. Mulvey, Transitivity and the center for maps of the circle, Ergodic Theory Dynamical Systems 6 (1986), 1-8.

[G] F. Gantmacher, The theory of matrices, vol. 2, Chelsea, New York, 1959.

[MS] M. Misiurewicz and W. Szlenk, Entropy of piecewise monotone mappings, Studia Math. 67 (1980), 45-63.

[N] Z. Nitecki, Topological dynamics on the interval, Ergodic theory and dynamical systems. II (College Park, Md., 1979-80), pp. 1-73, Progress in Math., vol. 21, Birkhauser, Boston, Mass., 1982.

[P] W. Parry, Symbolic dynamics and transformations of the unit interval, Trans. Amer. Math. Soc. 122 (1966), 368-378.

[RZ] H. Russmann and E. Zehnder, On a normal form of symmetric maps of $[0,1]$, Comm. Math. Phys. 72 (1980), 49-53.

[Sa] A. N. Sarkovskiǐ, Co-existence of cycles of a continuous mapping of the line into itself, Ukrain Math. Z. 16 (1964), 61-71. (Russian, English summary)

[St] P. Stefan, A theorem of Sarkovskit on the existence of periodic orbits of continuous endomorphisms of the real line, Comm. Math. Phys. 54 (1977), 237-248.

[SU] P. Stein and S. Ulam, Nonlinear transformation studies on electronic computers, Dissertationes Math. (Rozprawy Mat.) 39 (1964), 1-66.

Department of Mathematics, University of Florida, Gainesville, Florida 32611

Department of Mathematics, Wesleyan University, Middletown, ConnectiCUT 06457 